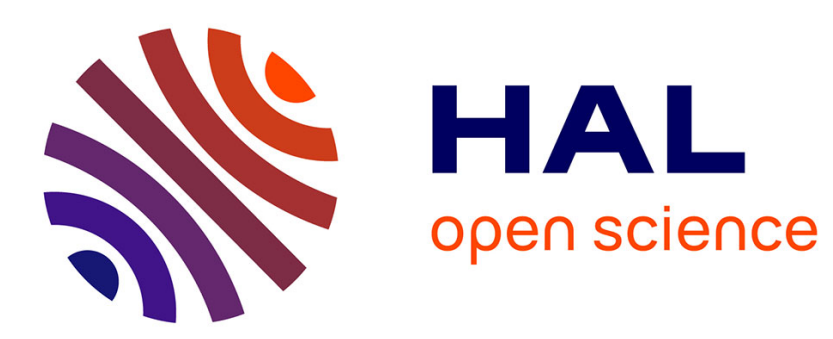

\title{
The $x$-coordinates of Pell equations and sums of two Fibonacci numbers II
}

\author{
Mahadi Ddamulira, Florian Luca
}

\section{To cite this version:}

Mahadi Ddamulira, Florian Luca. The $x$-coordinates of Pell equations and sums of two Fibonacci numbers II. Proceedings Mathematical Sciences, In press. hal-02266438

\section{HAL Id: hal-02266438 \\ https://hal.science/hal-02266438}

Submitted on 20 Aug 2019

HAL is a multi-disciplinary open access archive for the deposit and dissemination of scientific research documents, whether they are published or not. The documents may come from teaching and research institutions in France or abroad, or from public or private research centers.
L'archive ouverte pluridisciplinaire HAL, est destinée au dépôt et à la diffusion de documents scientifiques de niveau recherche, publiés ou non, émanant des établissements d'enseignement et de recherche français ou étrangers, des laboratoires publics ou privés. 


\title{
The $x$-coordinates of Pell equations and sums of two Fibonacci numbers II
}

\author{
MAHADI DDAMULIRA ${ }^{* 1}$ and FLORIAN LUCA ${ }^{2,3,4}$ \\ ${ }^{1}$ Institute of Analysis and Number Theory, Graz University of Technology, \\ Kopernikusgasse 24/II, A-8010 Graz, Austria \\ ${ }^{2}$ School of Mathematics, University of the Witwatersrand, Johannesburg, South Africa \\ ${ }^{3}$ Research Group in Algebraic Structures and Applications, King Abdulaziz University, \\ Jeddah, Saudi Arabia \\ ${ }^{4}$ Department of Mathematics, University of Ostrava, 30 Dubna 22, 70103 Ostrava 1, \\ Czech Republic \\ *Corresponding author. \\ Email: mddamulira@tugraz.at; mahadi@aims.edu.gh; Florian.Luca@wits.ac.za \\ MS received xx xxxxxxx 2019; revised xx xxxxxxxx 20xx
}

\begin{abstract}
Let $\left\{F_{n}\right\}_{n \geq 0}$ be the sequence of Fibonacci numbers defined by $F_{0}=0$, $F_{1}=1$ and $F_{n+2}=F_{n+1}+F_{n}$ for all $n \geq 0$. In this paper, for an integer $d \geq 2$ which is square-free, we show that there is at most one value of the positive integer $x$ participating in the Pell equation $x^{2}-d y^{2}= \pm 4$ which is a sum of two Fibonacci numbers, with a few exceptions that we completely characterize.
\end{abstract}

Keywords. Fibonacci number; Pell equation; linear form in logarithm; reduction method.

2010 Mathematics Subject Classification. 11B39, 11D45, 11D61, 11J86.

\section{Introduction}

Let $\left\{F_{n}\right\}_{n \geq 0}$ be the sequence of Fibonacci numbers given by

$$
F_{0}=0, F_{1}=1 \text { and } F_{n+2}=F_{n+1}+F_{n} \text { for all } n \geq 0 .
$$

The Fibonacci sequence is sequence $A 000045$ on the Online Encyclopedia of Integer Sequences (OEIS). The first few terms of this sequence are

$$
\left\{F_{n}\right\}_{n \geq 0}=0,1,1,2,3,5,8,13,21,34,55,89,144,233,377,610,987,1597,2584, \ldots .
$$

In this paper, we let $U:=\left\{F_{n}+F_{m}: n \geq m \geq 0\right\}$ be the sequence of sums of two Fibonacci numbers. The first few members of $U$ are

$$
U=\{0,1,2,3,4,5,6,7,8,9,10,11,13,14,15,16,18,21,22,23,24,26,29,34,35, \ldots\} .
$$

Let $d \geq 2$ be a positive integer which is not a square. It is well known that the Pell equation

$$
x^{2}-d y^{2}= \pm 4,
$$

(C) Indian Academy of Sciences 
has infinitely many positive integer solutions $(x, y)$. By putting $\left(x_{1}, y_{1}\right)$ for the smallest positive solutions to (1), all solutions are of the forms $\left(x_{k}, y_{k}\right)$ for some positive integer $k$, where

$$
\frac{x_{k}+y_{k} \sqrt{d}}{2}=\left(\frac{x_{1}+y_{1} \sqrt{d}}{2}\right)^{k} \quad \text { for all } \quad k \geq 1,
$$

Furthermore, the sequence $\left\{x_{k}\right\}_{k \geq 1}$ is binary recurrent. In fact, the following formula

$$
x_{k}=\left(\frac{x_{1}+y_{1} \sqrt{d}}{2}\right)^{k}+\left(\frac{x_{1}-y_{1} \sqrt{d}}{2}\right)^{k},
$$

holds for all positive integers $k$.

Recently, Gómez and Luca [2] studied the Diophantine equation

$$
x_{k}=F_{m}+F_{n}, \quad \text { with } \quad n \geq m \geq 0 \text {, }
$$

where $x_{k}$ are the $x$-coordinates of the solutions of the Pell equation $x^{2}-d y^{2}= \pm 1$ for some positive integer $k$ and $\left\{F_{n}\right\}_{n \geq 0}$ is the sequence of Fibonacci numbers. They proved that for each square free integer $d \geq 2$, there is at most one positive integer $k$ such that $x_{k}$ admits the representation (3) for some nonnegative integers $0 \leq m \leq n$, except for $d \in$ $\{2,3,5,11,30\}$. Furthermore, they explicitly stated all the solutions for these exceptional cases.

In the same spirit, Bravo et al. [1] studied the Diophantine equation

$$
x_{k}=T_{m}+T_{n}, \quad \text { with } n \geq m \geq 0 .
$$

where $x_{k}$ are the $x$-coordinates of the solutions of the Pell equation $x^{2}-d y^{2}= \pm 1$ for some positive integer $k$ and $\left\{T_{n}\right\}_{n \geq 0}$ is the sequence of Tribonacci numbers given by $T_{0}=0, T_{1}=1=T_{2}$ and $T_{n+3}=T_{n+2}+T_{n+1}+T_{n}$ for all $n \geq 0$. They proved that for each square free integer $d \geq 2$, there is at most one positive integer $k$ such that $x_{k}$ admits the representation (3) for some nonnegative integers $0 \leq m \leq n$, except for $d \in\{2,3,5,15,26\}$. Furthermore, they explicitly stated all the solutions for these exceptional cases. Several other related problems have been studied where $x_{k}$ belongs to some interesting positive integer sequences. For example, see [2, 5, 6, 7, 9, 11, 12, 13, 14, 15].

\section{Main Result}

In this paper, we study a problem related to that of Gómez and Luca [2], but for the Pell equation (1) instead of $x^{2}-d y^{2}= \pm 1$. Before formulating our main theorem, let us notice that our problem is a bit different from the previous ones in that there are infinitely many $d$ 's such that the equation

$$
x_{k}=F_{n}+F_{m} \quad \text { with } \quad n \geq m \geq 0
$$

has at least two solutions $(m, n, k)$. Indeed, take $d=5 u^{2}$ with some integer $u \geq 1$. Then positive solutions integer solutions $(x, y)$ to the Diophantine equation

$$
x^{2}-d y^{2}= \pm 4
$$

correspond to positive integer solutions $(X, Y):=(x, u y)$ to $X^{2}-5 Y^{2}= \pm 4$. It is wellknown that these are parametrised by $(X, Y)=\left(L_{n}, F_{n}\right)$, where $\left\{L_{n}\right\}_{n \geq 0}$ is the Lucas companion of the Fibonacci sequence given by $L_{0}=2, L_{1}=1$ and $L_{n+2}=L_{n+1}+L_{n}$ for $n \geq 0$. Furthermore, in this case $L_{n}^{2}-5 F_{n}^{2}=4(-1)^{n}$. Thus, the sign in the right-hand side is given by the parity of $n$. Now say $u$ is fixed and $F_{n}=u y$. Then $y=F_{n} / u$ and $F_{n}$ must be a multiple of $u$. It is well-known that $u \mid F_{n}$ is and only if $z(u) \mid n$, where 
$z(u)$ is the smallest positive integer $\ell$ such that $u \mid F_{\ell}$. This always exists and is called the index of appearance of $u$ in the Fibonacci sequence. We conclude that for $d=5 u^{2}$, we have $\left(x_{k}, y_{k}\right)=\left(L_{z(u) k}, F_{z(u) k} / u\right)$. In particular, $x_{k}=L_{n_{k}}$ for some positive integer $n_{k}$. Since $L_{n}=F_{n+1}+F_{n-1}$ holds for all $n \geq 1$, it follows that for all values of $k, x_{k}$ is a sum of two Fibonacci numbers. This gives an infinite parametric family of exceptions which dod not exist in any of the cases treated by others.

The main aim of this paper is to prove the following result.

Theorem 1. Let $d \geq 2$ be an integer which is not a square. If $d \neq 5 \square$, then is at most one positive integer $k$ such that $x_{k}$ admits a representation as

$$
x_{k}=F_{n}+F_{m}
$$

for some nonnegative integers $0 \leq m \leq n$, except when $d \in\{2,3,7,21,26\}$.

For the exceptional values of $d$ listed in Theorem 1 , all solutions $(k, n, m)$ are listed at the end of the paper. The main tools used in this paper are the lower bounds for linear forms in logarithms of algebraic numbers and the Baker-Davenport reduction procedure, as well as the elementary properties of Fibonacci numbers and solutions to Pell equations.

\section{Preliminary results}

\subsection{The Fibonacci sequence}

Here, we recall some important properties of the Fibonacci sequence $\left\{F_{n}\right\}_{n \geq 0}$. The characteristic equation

$$
x^{2}-x-1=0
$$

has roots $\alpha$ and $\beta$, where

$$
\alpha=\frac{1+\sqrt{5}}{2} \text { and } \beta=\frac{1-\sqrt{5}}{2} \text {. }
$$

The Binet formula for its geneneral terms is given by

$$
F_{n}=\frac{\alpha^{n}-\beta^{n}}{\sqrt{5}} \quad \text { for all } n \geq 0 .
$$

Furthermore, by induction, we can prove that

$$
\alpha^{n-2} \leq F_{n} \leq \alpha^{n-1} \quad \text { holds for all } \quad n \geq 1 .
$$

Let $\left\{L_{n}\right\}_{n \geq 0}$ be the sequence of Lucas numbers defined by $L_{0}=2, L_{1}=1$ and $L_{n+2}=$ $L_{n+1}+L_{n}$ for all $n \geq 0$. For all nonnegative integers $n$, the following hold.

$$
L_{n}=F_{n-1}+F_{n+1}
$$

and

$$
L_{n}^{2}-5 F_{n}^{2}=4(-1)^{n}
$$

The above identities will be useful in the next parts of this paper.

\subsection{Linear forms in logarithms}

Let $\eta$ be an algebraic number of degree $d$ with minimal primitive polynomial over the integers

$$
a_{0} x^{d}+a_{1} x^{d-1}+\cdots+a_{d}=a_{0} \prod_{i=1}^{d}\left(x-\eta^{(i)}\right),
$$


where the leading coefficient $a_{0}$ is positive and the $\eta^{(i)}$ 's are the conjugates of $\eta$. Then the logarithmic height of $\eta$ is given by

$$
h(\eta):=\frac{1}{d}\left(\log a_{0}+\sum_{i=1}^{d} \log \left(\max \left\{\left|\eta^{(i)}\right|, 1\right\}\right)\right) .
$$

In particular, if $\eta=p / q$ is a rational number with $\operatorname{gcd}(p, q)=1$ and $q>0$, then $h(\eta)=\log \max \{|p|, q\}$. The following are some of the properties of the logarithmic height function $h(\cdot)$, which will be used in the next sections of this paper without reference:

$$
\begin{aligned}
h\left(\eta_{1} \pm \eta_{2}\right) & \leq h\left(\eta_{1}\right)+h\left(\eta_{2}\right)+\log 2, \\
h\left(\eta_{1} \eta_{2}^{ \pm 1}\right) & \leq h\left(\eta_{1}\right)+h\left(\eta_{2}\right), \\
h\left(\eta^{s}\right) & =|s| h(\eta) \quad(s \in \mathbb{Z}) .
\end{aligned}
$$

We start by recalling the result of Bugeaud, Mignotte and Siksek ([3], Theorem 9.4, pp. 989), which is a modified version of the result of Matveev [16]. This result is one of our main tools in this paper.

Theorem 2. Let $\eta_{1}, \ldots, \eta_{t}$ be positive real numbers in number field $\mathbb{K} \subseteq \mathbb{R}$ of degree $D_{\mathbb{K}}$, $b_{1}, \ldots, b_{t}$ be nonzero integers, and assume that

$$
\Lambda:=\eta_{1}^{b_{1}} \cdots \eta_{t}^{b_{t}}-1,
$$

is nonzero. Then

$$
\log |\Lambda|>-1.4 \times 30^{t+3} \times t^{4.5} \times D_{\mathbb{K}}^{2}\left(1+\log D_{\mathbb{K}}\right)(1+\log B) A_{1} \cdots A_{t},
$$

where

$$
B \geq \max \left\{\left|b_{1}\right|, \ldots,\left|b_{t}\right|\right\}
$$

and

$$
A_{i} \geq \max \left\{D_{\mathbb{K}} h\left(\eta_{i}\right),\left|\log \eta_{i}\right|, 0.16\right\}, \quad \text { for all } \quad i=1, \ldots, t .
$$

\subsection{Reduction procedure}

During the calculations, we get upper bounds on our variables which are too large, thus we need to reduce them. To do so, we use some results from the theory of continued fractions.

For the treatment of linear forms homogeneous in two integer variables, we use the well-known classical result in the theory of Diophantine approximation.

Lemma 3. Let $\tau$ be an irrational number, $\frac{p_{0}}{q_{0}}, \frac{p_{1}}{q_{1}}, \frac{p_{2}}{q_{2}}, \ldots$ be all the convergents of the continued fraction of $\tau$ and $M$ be a positive integer. Let $N$ be a nonnegative integer such that $q_{N}>M$. Then putting $a(M):=\max \left\{a_{i}: i=0,1,2, \ldots, N\right\}$, the inequality

$$
\left|\tau-\frac{r}{s}\right|>\frac{1}{(a(M)+2) s^{2}},
$$

holds for all pairs $(r, s)$ of positive integers with $0<s<M$.

For a nonhomogeneous linear form in two integer variables, we use a slight variation of a result due to Dujella and Pethő (see [8], Lemma 5a). For a real number $X$, we write $\| X \mid:=\min \{|X-n|: n \in \mathbb{Z}\}$ for the distance from $X$ to the nearest integer. 
Lemma 4. Let $M$ be a positive integer, $\frac{p}{q}$ be a convergent of the continued fraction of the irrational number $\tau$ such that $q>6 M$, and $A, B, \mu$ be some real numbers with $A>0$ and $B>1$. Let further $\varepsilon:=\|\mu q\|-M\|\tau q\|$. If $\varepsilon>0$, then there is no solution to the inequality

$$
0<|u \tau-v+\mu|<A B^{-w}
$$

in positive integers $u, v$ and $w$ with

$$
u \leq M \quad \text { and } \quad w \geq \frac{\log (A q / \varepsilon)}{\log B} .
$$

At various occasions, we need to find a lower bound for linear forms in logarithms with bounded integer coefficients in three and four variables. In this case we use the LLL algorithm that we describe below. Let $\tau_{1}, \tau_{2}, \ldots \tau_{t} \in \mathbb{R}$ and the linear form

$$
x_{1} \tau_{1}+x_{2} \tau_{2}+\cdots+x_{t} \tau_{t} \text { with }\left|x_{i}\right| \leq X_{i} .
$$

We put $X:=\max \left\{X_{i}\right\}, C>(t X)^{t}$ and consider the integer lattice $\Omega$ generated by

$$
\mathbf{b}_{j}:=\mathbf{e}_{j}+\left\lfloor C \tau_{j}\right\rceil \text { for } \quad 1 \leq j \leq t-1 \quad \text { and } \quad \mathbf{b}_{t}:=\left\lfloor C \tau_{t}\right\rceil \mathbf{e}_{t},
$$

where $C$ is a sufficiently large positive constant.

Lemma 5. Let $X_{1}, X_{2}, \ldots, X_{t}$ be positive integers such that $X:=\max \left\{X_{i}\right\}$ and $C>(t X)^{t}$ is a fixed sufficiently large constant. With the above notation on the lattice $\Omega$, we consider a reduced base $\left\{\boldsymbol{b}_{i}\right\}$ to $\Omega$ and its associated Gram-Schmidt orthogonalization base $\left\{\boldsymbol{b}_{i}^{*}\right\}$. We set

$$
c_{1}:=\max _{1 \leq i \leq t} \frac{\left\|\boldsymbol{b}_{1}\right\|}{\left\|\boldsymbol{b}_{i}^{*}\right\|}, \quad \theta:=\frac{\left\|\boldsymbol{b}_{1}\right\|}{c_{1}}, \quad Q:=\sum_{i=1}^{t-1} X_{i}^{2} \quad \text { and } \quad R:=\frac{1}{2}\left(1+\sum_{i=1}^{t} X_{i}\right) .
$$

If the integers $x_{i}$ are such that $\left|x_{i}\right| \leq X_{i}$, for $1 \leq i \leq t$ and $\theta^{2} \geq Q+R^{2}$, then we have

$$
\left|\sum_{i=1}^{t} x_{i} \tau_{i}\right| \geq \frac{\sqrt{\theta^{2}-Q}-R}{C}
$$

For the proof and further details, we refer the reader to the book of Cohen. (Proposition 2.3.20 in ([4], pp. 58-63).

Finally, the following lemma is also useful. It is Lemma 7 in [10].

Lemma 6. If $r \geqslant 1, H>\left(4 r^{2}\right)^{r}$ and $H>L /(\log L)^{r}$, then

$$
L<2^{r} H(\log H)^{r}
$$

\section{Proof of Theorem 1}

Let $\left(x_{1}, y_{1}\right)$ be the smallest positive integer solution to the Pell quation (1). We Put

$$
\delta:=\frac{x_{1}+y_{1} \sqrt{d}}{2} \text { and } \sigma=\frac{x_{1}-y_{1} \sqrt{d}}{2} .
$$

From which we get that

Then

$$
\delta \cdot \sigma=\frac{x_{1}^{2}-d y_{1}^{2}}{4}=: \epsilon, \quad \text { where } \quad \epsilon \in\{ \pm 1\} .
$$

$$
x_{k}=\delta^{k}+\sigma^{k} \text {. }
$$


Since $\delta \geq \alpha$, it follows that the estimate

$$
\frac{\delta^{k}}{\alpha}<x_{k}<\alpha \delta^{k} \quad \text { holds for all } \quad k \geq 1 \text {. }
$$

We assume that $\left(k_{1}, n_{1}, m_{1}\right)$ and $\left(k_{2}, n_{2}, m_{2}\right)$ are triples of integers such that

$$
x_{k_{1}}=F_{n_{1}}+F_{m_{1}} \text { and } x_{k_{2}}=F_{n_{2}}+F_{m_{2}}
$$

We assume that $1 \leq k_{1}<k_{2}$.

Furthermore, by the well-known properties of solutions to Pell equations, we may assume that $\operatorname{gcd}\left(k_{1}, k_{2}\right)=1$. That is, if $\operatorname{gcd}\left(k_{1}, k_{2}\right)=\ell$, we then write $k_{1}=k_{1}^{\prime} \ell, k_{2}=k_{2}^{\prime} \ell$. We replace $d$ by $d^{\prime}:=d y_{\ell}^{2}$. Then the smallest solution $\left(x_{1}^{\prime}, y_{1}^{\prime}\right)$ of the Pell equation $x^{\prime 2}-d^{\prime} y^{\prime 2}= \pm 4$ is $\left(x_{\ell}, 1\right)$. Furthermore, $x_{k_{1}^{\prime}}^{\prime}=x_{k_{1}}$ and $x_{k_{2}^{\prime}}^{\prime}=x_{k_{2}}$. This justifies our claim that we may assume that $\operatorname{gcd}\left(k_{1}, k_{2}\right)=1$.

Next, $F_{1}=F_{2}=1$, so it follows that we may assume that $m_{i} \geq 2$ if $m_{i} \neq 0$. Thus, we either have $\left(m_{i}, n_{i}\right)=\left(0, n_{i}\right)$ with $n_{i} \geq 2$ or $2 \leq m_{i} \leq n_{i}$. If $m=n$, then $F_{m}+F_{n}=2 F_{n}$. If $n=2$, then $2 F_{n}=F_{3}$. Otherwise, $2 F_{n}=F_{n+1}+F_{n-2}$ and $n \geq 3$. Thus, we may always assume that $m_{i}<n_{i}$ for $i=1,2$. Finally, if $m=n-1$, then $F_{m}+F_{n}=F_{n-1}+F_{n}=F_{n+1}$. Thus, if $2 \leq m_{i}<n_{i}$, we may assume that $m_{i}$ and $n_{i}$ are not consecutive. In particular, either $\left(m_{i}, n_{i}\right)=(0,2)$. or $n_{i} \geq 3$. Let us treat the case $\left(m_{i}, n_{i}\right)=(0,2)$. In this case, $x_{k}=F_{0}^{2}+F_{2}^{2}=1$. Thus, $1^{2}-d y^{2}= \pm 4$. The only possibility is the sign - in the right-hand side, for which $d=5$, a case which we have excluded.

Thus, $n_{i} \geq 3$ for $i=1,2$.

Using the inequalities (6) and (15), we get from (16) that

$$
\frac{\delta^{k}}{\alpha} \leq x_{k}=F_{n}+F_{m} \leq F_{n}+F_{n-2} \leq \alpha^{n} \quad \text { and } \quad \alpha^{n-2} \leq F_{n}+F_{m}=x_{k} \leq \alpha \delta^{k} .
$$

The above inequalities give

$$
(n-3) \log \alpha<k \log \delta<(n+1) \log \alpha .
$$

Dividing through by $\log \alpha$ and setting $c_{2}:=1 / \log \alpha$, we get that

$$
-3<c_{2} k \log \delta-n<1,
$$

and since $\alpha^{3 / 2}>2$, we get

$$
\left|n-c_{2} k \log \delta\right|<3 \text {. }
$$

Furthermore, $k \leq n$, for if not, we would then get that

$$
\alpha^{n+1} \leq \delta^{n+1} \leq \delta^{k}<\alpha^{n+1},
$$

a contradiction. Besides, given that $k_{1}<k_{2}$, we have by (6) and (16) that

$$
\alpha^{n_{1}-2} \leq F_{n_{1}} \leq F_{n_{1}}+F_{m_{1}}=x_{k_{1}}<x_{k_{2}}=F_{n_{2}}+F_{m_{2}} \leq F_{n_{2}}+F_{n_{2}-2} \leq \alpha^{n_{2}-1}+\alpha^{n_{2}-3}<\alpha^{n_{2}} \text {. }
$$

Thus, we get that

$$
n_{1}<n_{2}+2 \text {. }
$$

\subsection{An inequality for $n$ and $k$}

Using the equations (5) and (14) and (16), we get

So,

$$
\delta^{k}+\sigma^{k}=F_{n}+F_{m}=\frac{\alpha^{n}-\beta^{n}}{\sqrt{5}}+\frac{\alpha^{m}-\beta^{m}}{\sqrt{5}} .
$$

$$
\delta^{k}-\frac{\alpha^{n}+\alpha^{m}}{\sqrt{5}}=-\sigma^{k}-\frac{\beta^{n}+\beta^{m}}{\sqrt{5}},
$$


and by (6), we have

$$
\begin{aligned}
\left|\delta^{k} \cdot \sqrt{5} \cdot \alpha^{-n}\left(1+\alpha^{m-n}\right)^{-1}-1\right| & \leq \frac{\sqrt{5}}{\delta^{k}\left(\alpha^{n}+\alpha^{m}\right)}+\frac{|\beta|^{n}+|\beta|^{m}}{\alpha^{n}+\alpha^{m}} \\
& \leq \frac{\sqrt{5} \alpha}{\alpha^{n}\left(\alpha^{n}+\alpha^{m}\right)}+\frac{1}{\alpha^{n+m}} \\
& \leq \frac{1}{\alpha^{n}}\left(\frac{\sqrt{5}}{\alpha^{n}+\alpha^{m}}+\frac{1}{\alpha^{m}}\right)<\frac{2}{\alpha^{n}}
\end{aligned}
$$

The numerator 1.5 above comes from the fact that $m \geq 0$ and $n \geq 3$. Thus, we have

Put

$$
\left|\delta^{k}(\sqrt{5}) \alpha^{-n}\left(1+\alpha^{m-n}\right)^{-1}-1\right|<\frac{2}{\alpha^{n}}
$$

and

$$
\Lambda_{1}:=\delta^{k}(\sqrt{5}) \alpha^{-n}\left(1+\alpha^{m-n}\right)^{-1}-1
$$

$$
\Gamma_{1}:=k \log \delta+\log (\sqrt{5})-n \log \alpha-\log \left(1+\alpha^{m-n}\right) .
$$

Since $\left|\Lambda_{1}\right|=\left|e^{\Gamma_{1}}-1\right|<\frac{1}{2}$ for $n \geq 3$ (because $n \geq 3$ and $\alpha^{3}>4$, so $2 / \alpha^{n} \leq 2 / \alpha^{3}<1 / 2$ ), and since the inequality $|y|<2\left|e^{y}-1\right|$ holds for all $y \in\left(-\frac{1}{2}, \frac{1}{2}\right)$, it follows that $e^{\left|\Gamma_{1}\right|}<2$ and so

Thus, we get that

$$
\left|\Gamma_{1}\right|<e^{\left|\Gamma_{1}\right|}\left|e^{\Gamma_{1}}-1\right|<\frac{4}{\alpha^{n}} .
$$

$$
\left|k \log \delta+\log (\sqrt{5})-n \log \alpha-\log \left(1+\alpha^{m-n}\right)\right|<\frac{4}{\alpha^{n}} .
$$

We apply Theorem 2 on the left-hand side of (19) with the data:

$$
\begin{aligned}
t:=4, \quad \eta_{1}:=\delta, \quad \eta_{2}:=\sqrt{5}, \quad \eta_{3}:=\alpha, \quad \eta_{4}:=1+\alpha^{m-n}, \\
b_{1}:=k, \quad b_{2}:=1, \quad b_{3}:=-n, \quad b_{4}:=-1 .
\end{aligned}
$$

Furthermore, we take the number field $\mathbb{K}:=\mathbb{Q}(\sqrt{d}, \alpha)$ which has degree $D_{\mathbb{K}}:=4$. Since $\max \{1, k, n\} \leq n$, we take $B:=n$. First we note that the left-hand side of (19) is non-zero, since otherwise,

$$
\delta^{k}=\frac{1}{\sqrt{5}}\left(\alpha^{n}+\alpha^{m}\right)
$$

The left-hand side belongs to the quadratic field $\mathbb{Q}(\sqrt{d})$ and is not rational while the righthand side belongs to the field $\mathbb{Q}(\sqrt{5})$. This is not possible since $d \neq 5$. Thus, $\Lambda_{1} \neq 0$ and we can apply Theorem 2 .

We have $h\left(\eta_{1}\right)=h(\delta)=\frac{1}{2} \log \delta, h\left(\eta_{2}\right)=h(\sqrt{5})=\frac{1}{2} \log 5$ and $h\left(\eta_{3}\right)=h(\alpha)=\frac{1}{2} \log \alpha$. On the other hand,

$$
\begin{aligned}
h\left(\eta_{4}\right) & =h\left(1+\alpha^{m-n}\right) \leq h(1)+h\left(\alpha^{m-n}\right)+\log 2 \\
& =(n-m) h(\alpha)+\log 2=\frac{1}{2}(n-m) \log \alpha+\log 2 .
\end{aligned}
$$

Thus, we can take

$$
A_{1}:=2 \log \delta, \quad A_{2}:=2 \log 5, \quad A_{3}:=2 \log \alpha, \quad A_{4}:=2(n-m) \log \alpha+4 \log 2 .
$$

Now, Theorem 2 tells us that

$$
\begin{aligned}
\log \left|\Lambda_{1}\right|> & -1.4 \times 30^{7} \times 4^{4.5} \times 4^{2}(1+\log 4)(1+\log n)(2 \log \delta) \\
& \times(2 \log 5)(2 \log \alpha)(2(n-m) \log \alpha+4 \log 2) \\
> & -3.4 \times 10^{16}(n-m) \log n \log \delta \log \alpha .
\end{aligned}
$$

Comparing the above inequality with (19), we get

$$
n \log \alpha-\log 2<3.4 \times 10^{16}(n-m) \log n \log \delta \log \alpha .
$$


Hence, we get that

$$
n<3.5 \times 10^{16}(n-m) \log n \log \delta .
$$

We now return to the equation $x_{k}=F_{n}+F_{m}$ and rewrite it as

we obtain

$$
\delta^{k}-\frac{\alpha^{n}}{\sqrt{5}}=-\sigma^{k}-\frac{\beta^{n}}{\sqrt{5}}+F_{m}
$$

$$
\left|\delta^{k} \cdot \sqrt{5} \cdot \alpha^{-n}-1\right| \leq \frac{1}{\alpha^{n-m}}\left(\frac{1}{\alpha}+\frac{1}{\alpha^{n+m}}+\frac{\sqrt{5}}{\delta^{k} \alpha^{m}}\right)<\frac{2}{\alpha^{n-m}} .
$$

The numerator 2 in the right-hand side above comes from the fact that $m \geq 0, n \geq 3$, $\delta \geq 1+\sqrt{2}$. Put

$$
\Lambda_{2}:=\delta^{k} \cdot \sqrt{5} \cdot \alpha^{-n}-1 \quad \text { and } \quad \Gamma_{2}:=k \log \delta+\log (\sqrt{5})-n \log \alpha .
$$

If $n-m \geq 3$, then $2 / \alpha^{n-m} \leq 2 / \alpha^{3}<1 / 2$, so $\left|e^{\Lambda_{2}}-1\right|<\frac{1}{2}$. It follows that

$$
|k \log \delta+\log (\sqrt{5})-n \log \alpha|=\left|\Gamma_{2}\right|<e^{\left|\Lambda_{2}\right|}\left|e^{\Lambda_{2}}-1\right|<\frac{4}{\alpha^{n-m}} .
$$

We show that (23) holds for $n-m=2$ as well. Well, the case $(m, n)=(0,2)$ is not allowed (since $d=5)$. The case $(m, n)=(1,3)$ reduces to $(m, n)=(0,4)$ by our conventions, for which $x_{k}=F_{4}=3$, so $3^{2}-d y_{k}^{2}= \pm 4$, and since $d \neq 5$, we get $d=13$, so $\delta^{k}=(3+\sqrt{13}) / 2$. One checks that (23) holds in this particular case as well. In the same way, $(m, n)=(2,4)$ gives $x_{k}=F_{2}+F_{4}=4$, so $4^{2}-d y_{k}^{2}= \pm 4$ and since $d \neq 5$, we get $d y_{k}^{2}=12$. Thus, $\delta^{k}=2+\sqrt{3}$ and one checks that (23) holds in this case as well. Finally, for $m \geq 3$, we have $n+m=(m+2)+m \geq 8$, and now the factor 2 in the numerator of the right-hand side of (22) can be replaced by 1 . Since $1 / \alpha^{n-m} \leq 1 / \alpha^{2}<1 / 2$, it follows that (23) holds also in this case (even with the better numerator of 2 in the right-hand side instead of 4 ).

Furthermore, $\Lambda_{2} \neq 0$ (so $\Gamma_{2} \neq 0$ ), since $\delta^{k} \notin \mathbb{Q}(\alpha)$ by the previous argument.

We now apply Theorem 2 to the left-hand side of (22) with the data

$$
t:=3, \quad \eta_{1}:=\delta, \quad \eta_{2}:=\sqrt{5}, \quad \eta_{3}:=\alpha, \quad b_{1}:=k, \quad b_{2}:=1, \quad b_{3}:=-n .
$$

Thus, we have the same $A_{1}, A_{2}, A_{3}, B$ as before. Then, by Theorem 2, we conclude that $\log \left|\Lambda_{2}\right|>-2.4 \times 10^{14} \log n \log \delta \log \alpha$.

By comparing with (22), we get

$$
n-m<2.5 \times 10^{14} \log n \log \delta .
$$

We replace the bound (24) on $n-m$ in (21) and use the fact that $\delta^{k}<\alpha^{n+1}$, to obtain bounds on $n$ and $k$ in terms of $\log n$ and $\log \delta$. We now record what we have proved so far.

Lemma 7. Let $(k, n, m)$ be a solution to the equation $x_{k}=F_{n}+F_{m}$ with $0 \leq m \leq n$ and $d \neq 5 \square$, then

$$
k<4.2 \times 10^{30}(\log n)^{2} \log \delta \quad \text { and } n<8.8 \times 10^{30}(\log n)^{2}(\log \delta)^{2} .
$$

\subsection{Absolute bounds}

We recall that $(k, n, m)=\left(k_{i}, n_{i}, m_{i}\right)$, where $0 \leq m_{i} \leq n_{i}$, for $i=1,2$ and $1 \leq k_{1}<k_{2}$. Further, $n_{i} \geq 2$ for $i=1,2$. We return to (23) and write

$$
\left|\Gamma_{2}^{(i)}\right|:=\left|k_{i} \log \delta+\log (\sqrt{5})-n_{i} \log \alpha\right|<\frac{4}{\alpha^{n_{i}-m_{i}}}, \quad \text { for } \quad i=1,2 .
$$

We do a suitable cross product between $\Gamma_{2}^{(1)}, \Gamma_{2}^{(2)}$ and $k_{1}, k_{2}$ to eliminate the term involving $\log \delta$ in the above linear forms in logarithms:

$$
\begin{aligned}
\left|\Gamma_{3}\right| & :=\left|\left(k_{2}-k_{1}\right) \log (\sqrt{5})+\left(k_{1} n_{2}-k_{2} n_{1}\right) \log \alpha\right|=\left|k_{2} \Gamma_{2}^{(1)}-k_{1} \Gamma_{2}^{(2)}\right| \\
& \leq k_{2}\left|\Gamma_{2}^{(1)}\right|+k_{1}\left|\Gamma_{2}^{(2)}\right| \leq \frac{4 k_{2}}{\alpha^{n_{1}-m_{1}}}+\frac{4 k_{1}}{\alpha^{n_{2}-m_{2}}} \leq \frac{8 n_{2}}{\alpha^{\lambda}},
\end{aligned}
$$


where $\lambda:=\min _{1 \leq i \leq 2}\left\{n_{i}-m_{i}\right\}$.

We need to find an upper bound for $\lambda$. If $8 n_{2} / \alpha^{\lambda}>1 / 2$, we then get

$$
\lambda<\frac{\log \left(16 n_{2}\right)}{\log \alpha}<3 \log \left(16 n_{2}\right) .
$$

Otherwise, $\left|\Gamma_{3}\right|<\frac{1}{2}$, so

$$
\left|e^{\Gamma_{3}}-1\right|=\left|(\sqrt{5})^{k_{2}-k_{1}} \alpha^{k_{1} n_{2}-k_{2} n_{1}}-1\right|<2\left|\Gamma_{3}\right|<\frac{16 n_{2}}{\alpha^{\lambda}} .
$$

We apply Theorem 2 with the data: $t:=2, \eta_{1}:=\sqrt{5}, \eta_{2}:=\alpha, b_{1}:=k_{2}-k_{1}, b_{2}:=$ $k_{1} n_{2}-k_{2} n_{1}$. We take the number field $\mathbb{K}:=\mathbb{Q}(\alpha)$ and $D_{\mathbb{K}}:=2$. We begin by checking that $e^{\Gamma_{3}}-1 \neq 0$ (so $\Gamma_{3} \neq 0$ ). This is true because $\alpha$ and $\sqrt{5}$ are multiplicatively independent, since $\alpha$ is a unit in the ring of integers $\mathbb{Q}(\alpha)$ while the norm of $\sqrt{5}$ is $-5 \neq \pm 1$.

We note that $k_{2}-k_{1}<k_{2}<n_{2}$. Further, from (26), we have

$$
\left|k_{2} n_{1}-k_{1} n_{2}\right|<\left(k_{2}-k_{1}\right) \frac{\log (\sqrt{5})}{\log \alpha}+\frac{8 k_{2}}{\alpha^{\lambda} \log \alpha}<15 k_{2}<15 n_{2}
$$

given that $\lambda \geq 1$. So, we can take $B:=15 n_{2}$. By Theorem 2, with $A_{1}:=\log 5$ and $A_{2}:=\log \alpha$, we have that

$$
\begin{aligned}
\log \left|e^{\Gamma_{3}}-1\right| & >-1.4 \times 30^{5} \times 2^{4.5} \times 2 \times(1+\log 2)\left(1+\log \left(15 n_{2}\right)\right)(\log 5)(\log \alpha) \\
& >-1.7 \times 10^{10} \log \left(15 n_{2}\right) \log \alpha .
\end{aligned}
$$

By comparing this with (28), we get

$$
\lambda \log \alpha-\log \left(16 n_{2}\right)<1.7 \times 10^{10} \log \left(15 n_{2}\right) \log \alpha,
$$

which implies that

$$
\lambda<1.8 \times 10^{10} \log \left(15 n_{2}\right) .
$$

Note that (29) is better than (27), so (29) always holds. Without loss of generality, we can assume that $\lambda=n_{i}-m_{i}$, for $i=1,2$ fixed.

We set $\{i, j\}=\{1,2\}$ and return to (20) to replace $(k, n, m)=\left(k_{i}, n_{i}, m_{i}\right)$ :

$$
\left|\Gamma_{1}^{(i)}\right|=\left|k_{i} \log \delta+\log (\sqrt{5})-n_{i} \log \alpha-\log \left(1+\alpha^{m_{i}-n_{i}}\right)\right|<\frac{4}{\alpha^{n_{i}}},
$$

and also return to (23), replacing with $(k, n, m)=\left(k_{j}, n_{j}, m_{j}\right)$ :

$$
\left|\Gamma_{2}^{(j)}\right|=\left|k_{j} \log \delta+\log (\sqrt{5})-n_{j} \log \alpha\right|<\frac{4}{\alpha^{n_{j}-m_{j}}} .
$$

We perform a cross product on (30) and (31) in order to eliminate the term on $\log \delta$ :

$$
\begin{aligned}
\left|\Gamma_{4}\right| & :=\left|\left(k_{i}-k_{j}\right) \log (\sqrt{5})+\left(k_{j} n_{i}-k_{i} n_{j}\right) \log \alpha+k_{j} \log \left(1+\alpha^{m_{i}-n_{i}}\right)\right| \\
& =\left|k_{i} \Gamma_{2}^{(j)}-k_{j} \Gamma_{1}^{(i)}\right| \leq k_{i}\left|\Gamma_{2}^{(j)}\right|+k_{j}\left|\Gamma_{1}^{(i)}\right| \\
& <\frac{4 k_{i}}{\alpha^{n_{j}-m_{j}}}+\frac{4 k_{j}}{\alpha^{n_{i}}}<\frac{8 n_{2}}{\alpha^{\nu}}
\end{aligned}
$$

with $v:=\min \left\{n_{i}, n_{j}-m_{j}\right\}$. As before, we need to find an upper bound on $v$. If $8 n_{2} / \alpha^{v}>$ $1 / 2$, then we get

$$
v<\frac{\log \left(16 n_{2}\right)}{\log \alpha}<3 \log \left(16 n_{2}\right) .
$$


Otherwise, $\left|\Gamma_{4}\right|<1 / 2$, so we have

$$
\left|e^{\Gamma_{4}}-1\right|=\left|(\sqrt{5})^{k_{i}-k_{j}} \alpha^{k_{j} n_{i}-k_{i} n_{j}}\left(1+\alpha^{m_{i}-n_{i}}\right)^{k_{j}}-1\right| \leq 2\left|\Gamma_{4}\right|<\frac{16 n_{2}}{\alpha^{\nu}} .
$$

In order to apply Theorem 2 , first if $e^{\Gamma_{4}}=1$, we obtain

$$
(\sqrt{5})^{k_{j}-k_{i}}=\alpha^{k_{j} n_{i}-k_{i} n_{j}}\left(1+\alpha^{-\lambda}\right)^{k_{j}} .
$$

Let us show that the above equation is impossible. Since the right-hand side is an algebraic integer (because $\alpha$ is a unit), it follows that $k_{j}>k_{i}$. We take norms (in $\mathbb{Q}(\sqrt{5})$ ) and absolute values in both sides of (35). We then get

$$
5^{k_{j}-k_{i}}=\left(\left(1+\alpha^{\lambda}\right)\left(1+\beta^{\lambda}\right)\right)^{k_{j}}=\left\{\begin{array}{clll}
L_{\lambda}^{k_{j}} & \text { if } \lambda \equiv 1 & (\bmod 2) ; \\
L_{\lambda / 2}^{2 k_{j}} & \text { if } \lambda \equiv 0 & (\bmod 4) ; \\
\left(5 F_{\lambda / 2}^{2}\right)^{k_{j}} & \text { if } \lambda \equiv 2 & (\bmod 4) .
\end{array}\right.
$$

The above equation is impossible since the exponent of 5 in the left-hand side is positive and smaller than $k_{j}$, while in the right-hand side, ether it is at least $k_{j}($ if $\lambda \equiv 2(\bmod 4))$ or is $0($ if $\lambda \not \equiv 2(\bmod 4))$, because 5 never divides $L_{n}$ for any positive integer $n$. Hence, $e^{\Gamma_{4}} \neq 1$. We apply Theorem 2 with the data:

$$
\begin{gathered}
t:=3, \quad \eta_{1}:=\sqrt{5}, \quad \eta_{2}:=\alpha, \quad \eta_{3}:=1+\alpha^{-\lambda}, \\
b_{1}:=k_{i}-k_{j}, \quad b_{2}:=k_{j} n_{i}-k_{i} n_{j}, \quad b_{3}:=k_{j},
\end{gathered}
$$

We take $D_{\mathbb{K}}:=2, A_{1}:=\log 5, A_{2}:=\log \alpha, A_{3}:=\lambda \log \alpha+2 \log 2 \leq 2 \lambda \log \alpha$, and $B:=15 n_{2}$. By Theorem 2, we get that

$$
\begin{aligned}
\log \left|e^{\Gamma_{4}}-1\right| & >-1.4 \times 30^{6} \times 3^{4.5} \times 2(1+\log 2)\left(1+\log \left(15 n_{2}\right)\right)(\log 5)(\log \alpha)(2 \lambda \log \alpha) \\
& >-3.0 \times 10^{12} \lambda \log \left(15 n_{2}\right) \log \alpha .
\end{aligned}
$$

By comparing this with (34) together with the inequality (29), we get

$$
\begin{aligned}
v \log \alpha-\log \left(16 n_{2}\right) & <3.0 \times 10^{12} \lambda \log \left(15 n_{2}\right) \log \alpha, \\
v & :=\min \left\{n_{i}, n_{j}-m_{j}\right\}<3.2 \times 10^{12} \lambda \log \left(15 n_{2}\right)<5.8 \times 10^{22}\left(\log \left(15 n_{2}\right)\right)^{2} .
\end{aligned}
$$

Further, it also holds when the inequality (33) holds. So the above inequality holds in all cases. Note that the case $\{i, j\}=\{2,1\}$ leads to $n_{1}-m_{1} \leq n_{1} \leq n_{2}+2$ whereas $\{i, j\}=\{1,2\}$ lead to $v=\min \left\{n_{1}, n_{2}-m_{2}\right\}$. Hence, either the minimum is $n_{1}$, so

$$
n_{1}<5.8 \times 10^{22}\left(\log \left(15 n_{2}\right)\right)^{2}
$$

or the minimum is $n_{j}-m_{j}$ and from the inequality (29) we get that

$$
\max _{1 \leq j \leq 2}\left\{n_{j}-m_{j}\right\}<5.8 \times 10^{22}\left(\log \left(15 n_{2}\right)\right)^{2} \text {. }
$$

Next, we assume that we are in the case (39). We evaluate (30) in $i=1,2$ and make a suitable cross product to eliminate the term involving $\log \delta$ :

$$
\begin{aligned}
&\left|\Gamma_{5}\right|:=\mid\left(k_{1}-k_{2}\right) \log (\sqrt{5})+\left(k_{2} n_{1}-k_{1} n_{2}\right) \log \alpha \\
&+k_{2} \log \left(1+\alpha^{m_{1}-n_{1}}\right)-k_{1} \log \left(1+\alpha^{m_{2}-n_{2}}\right) \mid \\
&=\left|k_{1} \Gamma_{1}^{(2)}-k_{2} \Gamma_{1}^{(1)}\right| \leq k_{1}\left|\Gamma_{1}^{(2)}\right|+k_{2}\left|\Gamma_{1}^{(1)}\right|<\frac{8 n_{2}}{\alpha^{n_{1}}} .
\end{aligned}
$$

In the above inequality we used the inequality (18) to conclude that $\min \left\{n_{1}, n_{2}\right\} \geq n_{1}-3$ as well as the fact that $n_{i} \geq 3$ for $i=1,2$. Next, we apply a linear form in four logarithms to obtain an upper bound to $n_{1}$. As in the previous calculations, we pass from (40) to

$$
\left|e^{\Gamma_{5}}-1\right|=\left|(\sqrt{5})^{k_{1}-k_{2}} \alpha^{k_{2} n_{1}-k_{1} n_{2}}\left(1+\alpha^{m_{1}-n_{1}}\right)^{k_{2}}\left(1+\alpha^{m_{2}-n_{2}}\right)^{-k_{1}}-1\right|<\frac{16 n_{2}}{\alpha^{n_{1}}},
$$


which is implied by (40) except if $n_{1}$ is very small, say

$$
n_{1} \leq 3 \log \left(16 n_{2}\right) \text {. }
$$

Thus, we assume that (42) does not hold, therefore (41) holds. Then to apply Theorem 2, we first justify that $e^{\Gamma_{5}} \neq 1$. Otherwise,

$$
(\sqrt{5})^{k_{2}-k_{1}}=\alpha^{k_{2} m_{1}-k_{1} m_{2}}\left(1+\alpha^{n_{1}-m_{1}}\right)^{k_{2}}\left(1+\alpha^{n_{2}-m_{2}}\right)^{-k_{1}},
$$

We need to check that the equation (43) has no positive integer solutions. We let $\mathbb{K}:=$ $\mathbb{Q}(\sqrt{5})$. We use, as we did in (36), that for any positive integer $k$,

$$
N_{\mathbb{K} / \mathbb{Q}}\left(1+\alpha^{k}\right)= \begin{cases}L_{k}, & \text { if } \quad k \equiv 1(\bmod 2), \\ L_{k / 2}^{2}, & \text { if } k \equiv 0(\bmod 4), \\ 5 F_{k / 2}^{2}, & \text { if } k \equiv 2(\bmod 4) .\end{cases}
$$

Now, we assume that (43) holds and take norms and absolute values on both sides to get

$$
5^{k_{2}-k_{1}}=\left|N_{\mathbb{K} / \mathbb{Q}}(\sqrt{5})\right|^{k_{2}-k_{1}}=\left|N_{\mathbb{K} / \mathbb{Q}}(\alpha)\right|^{k_{2} m_{1}-k_{1} m_{2}} \frac{\left|N_{\mathbb{K} / \mathbb{Q}}\left(1+\alpha^{n_{1}-m_{1}}\right)\right|}{\left|N_{\mathbb{K} / \mathbb{Q}}\left(1+\alpha^{n_{2}-m_{2}}\right)\right|}=\frac{E_{n_{1}-m_{1}}^{k_{2}}}{E_{n_{2}-m_{2}}^{k_{1}}},
$$

where $E_{k} \in\left\{L_{k}, L_{k / 2}^{2}, 5 F_{k / 2}^{2}\right\}$ according to the residue class of $k$ modulo 4 . Since 5 divides the left-hand side above which is an integer, 5 divides the numerator of the right-hand side. Since $5 \nmid L_{m}$ for any $m$, it follows that $E_{n_{1}-m_{1}}=5 F_{\left(n_{1}-m_{1}\right) / 2}^{2}$. Then the exponent of 5 in the numerator of the right-hand side is at least $k_{2}>k_{2}-k_{1}$, we infer that 5 should also divide the denominator of the right-hand side meaning $E_{n_{2}-m_{2}}=5 F_{\left(n_{2}-m_{2}\right) / 2}^{2}$. But then we get

$$
F_{\left(n_{1}-m_{1}\right) / 2}^{2 k_{2}}=F_{\left(n_{2}-m_{2}\right) / 2}^{2 k_{1}} .
$$

Since $k_{2}>k_{1}$, we either have $F_{\left(n_{1}-m_{1}\right) / 2}<F_{\left(n_{2}-m_{2}\right) / 2}$ or both sides are 1 . The only distinct Fibonacci numbers which are multiplicatively dependent are 2 and $8=F_{6}$, but then $n_{2}-m_{2}=12$, so $E_{n_{2}-m_{2}}=L_{\left(n_{2}-m_{2}\right) / 2}^{2}$ (instead of $5 F_{\left(n_{2}-m_{2}\right) / 2}^{2}$ ), a contradiction. Hence, $F_{\left(n_{1}-m_{1}\right) / 2}=F_{\left(n_{2}-m_{2}\right) / 2}=1$ and since $\left(n_{i}-m_{i}\right) / 2$ is odd (in order for $E_{n_{i}-m_{i}}=5 F_{\left(n_{i}-m_{i}\right) / 2}^{2}$ to hold), we get $n_{i}-m_{i}=2$. Thus, $x_{k_{i}}=F_{n_{i}}+F_{n_{i}-2}=L_{n_{i}-1}$ for $i=1,2$.

Further, $1+\alpha^{n_{i}-m_{i}}=1+\alpha^{2}=\sqrt{5} \alpha$ for $i=1,2$ so (43) becomes

$$
\sqrt{5}^{k_{2}-k_{1}}=\alpha^{k_{2} m_{1}-k_{1} m_{2}}(\sqrt{5} \alpha)^{k_{2}}(\sqrt{5} \alpha)^{-k_{1}}=\sqrt{5}^{k_{2}-k_{1}} \alpha^{k_{2}\left(m_{1}+1\right)-k_{1}\left(m_{2}+1\right)} .
$$

We now get $k_{1}\left(n_{2}-1\right)=k_{2}\left(n_{1}-1\right)$ (because $n_{i}-1=m_{i}+1$ for $\left.i=1,2\right)$ and since $\operatorname{gcd}\left(k_{1}, k_{2}\right)=1$, we have $k_{1}=\left(n_{1}-1\right) / \ell$ and $k_{2}=\left(n_{2}-1\right) / \ell$ for some number $\ell$. Thus, $n_{1}-1=k_{1} \ell$ and $n_{2}-1=k_{2} \ell$. So, we get

$$
x_{k_{i}}=\delta^{k_{i}}+\sigma^{k_{i}}=\left(\alpha^{\ell}\right)^{k_{i}}+\left(\beta^{\ell}\right)^{k_{i}} \quad \text { for } \quad i=1,2 .
$$

Then

$$
\delta^{k_{i}}-\left(\alpha^{\ell}\right)^{k_{i}}=\left(\delta-\alpha^{\ell}\right)\left(\delta^{k_{i}-1}+\cdots+\left(\alpha^{\ell}\right)^{k_{i}-1}\right)=-\sigma^{k_{i}}+\left(\beta^{\ell}\right)^{k_{i}} .
$$

Assume now that $k_{2} \geq 3$. We then get

$$
\delta^{2}\left|\delta-\alpha^{\ell}\right|<\mid \delta-\alpha^{\ell}\left(\left(\delta^{k_{2}-1}+\cdots+\left(\alpha^{\ell}\right)^{k_{2}-1}\right) \leq \delta^{-k_{2}}+\left(\alpha^{\ell}\right)^{-k_{2}} .\right.
$$

Now $\delta \geq \alpha$, so $\delta^{-k_{2}}+\left(\alpha^{\ell}\right)^{-k_{2}} \leq 2 / \alpha^{3}$. Hence, $\delta^{2}\left|\delta-\alpha^{\ell}\right|<2 / \alpha^{3}$ giving $\left|\delta-\alpha^{\ell}\right|<2 / \alpha^{5}$. Thus, $\alpha^{\ell} \geq \delta-2 / \alpha^{5}$. Thus,

$$
\left(\alpha^{\ell}\right)^{-k_{2}} \leq\left(\alpha^{\ell}\right)^{-3} \leq\left(\delta-2 / \alpha^{5}\right)^{-3}<2 \delta^{-3},
$$


where we used the fact that

$$
\left(\frac{\delta}{\delta-2 / \alpha^{5}}\right)^{3}>2,
$$

which follows because $\delta>\alpha$ and $\alpha /\left(\alpha-2 / \alpha^{5}\right)<2^{1 / 3}$. We thus get that

$$
\delta^{2}\left|\delta-\alpha^{\ell}\right| \leq \delta^{-k_{2}}+\left(\alpha^{\ell}\right)^{-k_{2}} \leq 3 \delta^{-3},
$$

giving $\left|\delta-\alpha^{\ell}\right|<3 \delta^{-5}$. Assume $\delta \neq \alpha^{\ell}$. Then $\delta-\alpha^{\ell}$ is an algebraic integer of degree at most 4 and its conjugates are among $\sigma-\alpha^{\ell}, \sigma-\beta^{\ell}$ and $\delta-\beta^{\ell}$. These have absolute values at most $1 / \delta+\alpha^{\ell}<\delta+1 / \delta+3 / \delta^{5} \leq \delta+\left(1 / \alpha+3 / \alpha^{5}\right)<\delta+1,2$ and $\delta+1$ respectively. Computing the norm of the algebraic integer $\delta-\alpha^{\ell}$, we get

$$
1 \leq\left|N_{\mathbb{K} / \mathbb{Q}}\left(\delta-\alpha^{\ell}\right)\right| \leq\left(3 \delta^{-5}\right)\left(2(\delta+1)^{2}\right),
$$

giving $\delta<2.31$. Here, $\mathbb{K}=\mathbb{Q}(\sqrt{d}, \sqrt{5})$. The only value of $\delta<2.31$ is $\alpha$ (the next value of $\delta$ is $1+\sqrt{2}>2.4$ ). This shows that $k_{2} \geq 3$ is not possible. Thus, $k_{1}=1, k_{2}=2$, and so $n_{1}-1=\ell$ and $n_{2}-1=2 \ell$. So, we get

$$
x_{1}=L_{\ell} \quad \text { and } \quad x_{2}=L_{2 \ell} .
$$

Now putting

$$
x_{1}^{2}-d y_{1}^{2}=4 \epsilon,
$$

it follows that $x_{2}=x_{1}^{2}-2 \epsilon$, so $L_{2 \ell}^{2}=L_{\ell}^{2}-2 \epsilon$. Since in fact $L_{2 \ell}=L_{\ell}^{2}-2(-1)^{\ell}$, it follows that $\epsilon=(-1)^{\ell}$. Thus,

$$
L_{\ell}^{2}-d y_{1}^{2}=4(-1)^{\ell}
$$

and comparing it with the identity $L_{\ell}^{2}-5 F_{\ell}^{2}=4(-1)^{\ell}$, we get $d y_{1}^{2}=5 F_{\ell}^{2}$, so $d=5 u^{2}$ for some integer $u$ (which in this case is $F_{\ell} / y_{1}$ ), which is not the case. Thus, $e^{\Gamma_{5}} \neq 1$.

Thus, we apply Theorem 2 on the left-hand side of the inequalities (41) with the data

$$
\begin{gathered}
t:=4, \quad \eta_{1}:=\sqrt{5}, \quad \eta_{2}:=\alpha, \quad \eta_{3}:=1+\alpha^{m_{1}-n_{1}}, \quad \eta_{4}:=1+\alpha^{m_{2}-n_{2}}, \\
b_{1}:=k_{2}-k_{1}, \quad b_{2}:=k_{2} n_{1}-k_{1} n_{2}, \quad b_{3}:=k_{2}, \quad b_{4}:=-k_{1} .
\end{gathered}
$$

We take $D_{\mathbb{K}}:=2, a_{1}:=\log 5, A_{2}:=\log \alpha, A_{3}:=2\left(n_{1}-m_{1}\right) \log \alpha, A_{3}:=2\left(n_{2}-m_{2}\right) \log \alpha$, and $B:=15 n_{2}$. By Theorem 2, we get

$$
\begin{aligned}
\log \left|e^{\Gamma_{5}}-1\right|> & -1.4 \times 30^{7} \times 4^{4.5} \times 2^{2}(1+\log 2)\left(1+\log \left(15 n_{2}\right)\right)(\log 5)(\log \alpha) \\
& \times\left(2\left(n_{1}-m_{1}\right) \log \alpha\right)\left(2\left(n_{2}-m_{2}\right) \log \alpha\right) \\
> & -3.2 \times 10^{14}\left(n_{1}-m_{1}\right)\left(n_{2}-m_{2}\right) \log \left(15 n_{2}\right) \log \alpha .
\end{aligned}
$$

By comparing this with (41) together with the inequalities (29) and (39), we get

$$
\begin{aligned}
n_{1} & <3.3 \times 10^{14}\left(n_{1}-m_{1}\right)\left(n_{2}-m_{2}\right) \log \left(15 n_{2}\right) \\
& <3.5 \times 10^{47}\left(\log \left(15 n_{2}\right)\right)^{4} .
\end{aligned}
$$

In the above we used the facts that

$$
\min _{1 \leq i \leq 2}\left\{n_{i}-m_{i}\right\}<1.8 \times 10^{10} \log \left(15 n_{2}\right) \quad \text { and } \quad \max _{1 \leq i \leq 2}\left\{n_{i}-m_{i}\right\}<5.8 \times 10^{22}\left(\log \left(15 n_{2}\right)\right)^{2} .
$$

This was obtained under the assumption that the inequality (42) does not hold. If (42) holds, then so does (44). Thus, we have that inequality (44) holds provided that inequality 
(39) holds. Otherwise, inequality (38) holds which is a better bound than (44). Hence, conclude that (44) holds in all posibble cases.

We have,

$$
\log \delta \leq k_{1} \log \delta \leq\left(n_{1}+1\right) \log \alpha<1.7 \times 10^{49}\left(\log \left(15 n_{2}\right)\right)^{4} .
$$

By substituting this into $(25)$ we get $15 n_{2}<3.6 \times 10^{126}\left(\log \left(15 n_{2}\right)\right)^{10}$, and then, by Lemma 6 , with the data $r:=10, H:=3.6 \times 10^{126}$ and $L:=15 n_{2}$, we get that $15 n_{2}<1.6 \times 10^{154}$. This immediately gives that $n_{2}<1.1 \times 10^{153}$ and $n_{1}<5.6 \times 10^{57}$.

We record what we have proved.

Lemma 8. Let $\left(k_{i}, n_{i}, m_{i}\right)$ be a solution to $x_{k_{i}}=F_{n_{i}}+F_{m_{i}}$, with $0 \leq m_{i} \leq n_{i}$ for $i \in\{1,2\}$, $d \neq 5 \square$ and $1 \leq k_{1}<k_{2}$, then

$$
\max \left\{k_{1}, m_{1}\right\} \leq n_{1}<10^{58} \text { and } \max \left\{k_{2}, m_{2}\right\} \leq n_{2}<10^{154} \text {. }
$$

\section{Reducing the bounds for $n_{1}$ and $n_{2}$}

In this section we reduce the bounds for $n_{1}$ and $n_{2}$ given in Lemma 8 to cases that can be computationally treated. For this, we return to the inequalities for $\Gamma_{3}, \Gamma_{4}$ and $\Gamma_{5}$.

\subsection{The first reduction}

We divide through both sides of the inequality (26) by $\left(k_{2}-k_{1}\right) \log \alpha$. We get that

$$
\left|\frac{\log (\sqrt{5})}{\log \alpha}-\frac{k_{2} n_{1}-k_{1} n_{2}}{k_{2}-k_{1}}\right|<\frac{8 n_{2}}{\alpha^{\lambda}\left(k_{2}-k_{1}\right) \log \alpha} \quad \text { with } \quad \lambda:=\min _{1 \leq i \leq 2}\left\{n_{i}-m_{i}\right\} .
$$

We assume that $\lambda \geq 10$. Below we apply Lemma 3. We put $\tau:=\frac{\log (\sqrt{5})}{\log \alpha}$, which is irrational and compute its continued fraction

$$
\left[a_{0} ; a_{1}, a_{2}, \ldots\right]=[1 ; 1,2,19,2,9,1,1,3,1,9,1,2,6,1,1,1,5,1,14,29,1,2,1,4,2,1, \ldots]
$$

and its convergents

$$
\left[\frac{p_{0}}{q_{0}}, \frac{p_{1}}{q_{1}}, \frac{p_{2}}{q_{2}}, \ldots\right]=\left[1,2, \frac{5}{3}, \frac{97}{58}, \frac{199}{119}, \frac{1888}{1129}, \frac{2087}{1248}, \frac{3975}{2377}, \frac{14012}{8379}, \frac{17987}{10756}, \frac{175895}{105183}, \ldots\right] .
$$

Furthermore, we note that taking $M:=10^{154}$ (by Lemma 8), it follows that

$$
q_{297}>M>n_{2}>k_{2}-k_{1} \text { and } a(M):=\max \left\{a_{i}: 0 \leq i \leq 297\right\}=a_{170}=330 .
$$

Thus, by Lemma 3 , we have that

$$
\left|\tau-\frac{k_{2} n_{1}-k_{1} n_{2}}{k_{2}-k_{1}}\right|>\frac{1}{332\left(k_{2}-k_{1}\right)^{2}} .
$$

Hence, combining the inequalities (45) and (46), we obtain

$$
\alpha^{\lambda}<5519 n_{2}\left(k_{2}-k_{1}\right)<5.52 \times 10^{311}
$$

so $\lambda \leq 1491$. This was obtained under the assumption that $\lambda \geq 10$, Otherwise, $\lambda<10<$ 1491 holds as well.

Now, for each $n_{i}-m_{i}=\lambda \in[1,1491]$ we estimate a lower bound $\left|\Gamma_{4}\right|$, with

$$
\Gamma_{4}=\left(k_{i}-k_{j}\right) \log (\sqrt{5})+\left(k_{j} n_{i}-k_{i} n_{j}\right) \log \alpha+k_{j} \log \left(1+\alpha^{m_{i}-n_{i}}\right)
$$

given in the inequality (32), via the procedure described in Subsection 3.3 (LLL-algorithm). We recall that $\Gamma_{4} \neq 0$.

We apply Lemma 5 with the data:

$$
\begin{aligned}
t:=3, \quad \tau_{1} & :=\log (\sqrt{5}), \quad \tau_{2}:=\log \alpha, \quad \tau_{3}:=\log \left(1+\alpha^{-\lambda}\right), \\
x_{1} & :=k_{i}-k_{j}, \quad x_{2}:=k_{j} n_{i}-k_{i} n_{j}, \quad x_{3}:=k_{j} .
\end{aligned}
$$


We set $X:=15 \times 10^{154}$ as an upper bound to $\left|x_{i}\right|<15 n_{2}$ for all $i=1,2,3$, and $C:=(10 X)^{5}$. A computer in Mathematica search allows us to conclude, together with the inequality (32), that

$$
2 \times 10^{-653}<\min _{1 \leq \lambda \leq 1491}\left|\Gamma_{4}\right|<8 n_{2} \alpha^{-v}, \quad \text { with } \quad v:=\min \left\{n_{i}, n_{j}-m_{j}\right\}
$$

which leads to $v \leq 3855$. As we have noted before, $v=n_{1}$ (so $n_{1} \leq 3855$ ) or $v=n_{j}-m_{j}$.

Next, we suppose that $n_{j}-m_{j}=v \leq 3855$. Since $\lambda \leq 1491$, we have

$$
\lambda:=\min _{1 \leq i \leq 2}\left\{n_{i}-m_{i}\right\} \leq 1491 \text { and } \chi:=\max _{1 \leq i \leq 2}\left\{n_{i}-m_{i}\right\} \leq 3855 .
$$

Now, returning to the inequality (40) which involves

$$
\begin{aligned}
\Gamma_{5}:= & \left(k_{2}-k_{1}\right) \log (\sqrt{5})+\left(k_{2} n_{1}-k_{1} n_{2}\right) \log \alpha \\
& +k_{2} \log \left(1+\alpha^{m_{1}-n_{1}}\right)-k_{1} \log \left(1+\alpha^{m_{2}-n_{2}}\right) \neq 0,
\end{aligned}
$$

we use again the LLL-algorithm to estimate the lower bound for $\left|\Gamma_{5}\right|$ and thus, find a bound for $n_{1}$ that is better than the one given in Lemma 8 .

We distinguish the cases $\lambda<\chi$ and $\lambda=\chi$.

\subsection{The case $\lambda<\chi$.}

We take $\lambda \in[1,1491]$ and $\chi \in[\lambda+1,3855]$ and apply Lemma 5 with the data: $t:=4$,

$$
\begin{gathered}
\tau_{1}:=\log (\sqrt{5}), \quad \tau_{2}:=\log \alpha, \quad \tau_{3}:=\log \left(1+\alpha^{m_{1}-n_{1}}\right), \quad \tau_{4}:=\log \left(1+\alpha^{m_{2}-n_{2}}\right), \\
x_{1}:=k_{2}-k_{1}, \quad x_{2}:=k_{2} n_{1}-k_{1} n_{2}, \quad x_{3}:=k_{2}, \quad x_{4}:=-k_{1} .
\end{gathered}
$$

We also put $X:=15 \times 10^{154}$ and $C:=(20 X)^{9}$. After a computer search in Mathematica together with the inequality (40), we can confirm that

$$
10^{-1312}<\min _{\substack{1 \leq \lambda \leq 1491 \\ \lambda+1 \leq \chi \leq 3855}}\left|\Gamma_{5}\right|<8 n_{2} \alpha^{-n_{1}}
$$

This leads to the inequality

$$
\alpha^{n_{1}}<8 \times 10^{1312} n_{2} \text {. }
$$

Subsitituting for the bound $n_{2}$ given in Lemma 8 , we get that $n_{1} \leq 7019$.

\subsection{The case $\lambda=\chi$.}

In this case, we have

$$
\Gamma_{5}:=\left(k_{2}-k_{1}\right)\left(\log (1 / \sqrt{5})+\log \left(1+\alpha^{m_{1}-n_{1}}\right)\right)+\left(k_{2} n_{1}-k_{1} n_{2}\right) \log \alpha \neq 0 .
$$

We divide through the inequality 40 by $\left(k_{2}-k_{1}\right) \log \alpha$ to obtain

We now put

$$
\left|\frac{\left|\log (1 / \sqrt{5})+\log \left(1+\alpha^{m_{1}-n_{1}}\right)\right|}{\log \alpha}-\frac{k_{2} n_{1}-k_{1} n_{2}}{k_{2}-k_{1}}\right|<\frac{8 n_{2}}{\alpha^{n_{1}}\left(k_{2}-k_{1}\right) \log \alpha}
$$

$$
\tau_{\lambda}:=\frac{\left|\log (1 / \sqrt{5})+\log \left(1+\alpha^{-\lambda}\right)\right|}{\log \alpha}
$$

and compute its continued fractions $\left[a_{0}^{(\lambda)}, a_{1}^{(\lambda)}, a_{2}^{(\lambda)}, \ldots\right]$ and its convergents $\left[p_{0}^{(\lambda)} / q_{0}^{(\lambda)}, p_{1}^{(\lambda)} / q_{1}^{(\lambda)}, p_{2}^{(\lambda)} / q_{2}^{(\lambda)}, \ldots\right]$ for each $\lambda \in[1,1491]$. Furthermore, for each case we find an integer $t_{\lambda}$ such that $q_{t_{\lambda}}^{(\lambda)}>M:=10^{154}>n_{2}>k_{2}-k_{1}$ and calculate

$$
a(M):=\max _{1 \leq \lambda \leq 1491}\left\{a_{i}^{(\lambda)}: 0 \leq i \leq t_{\lambda}\right\} .
$$

A computer search in Mathematica reveals that for $\lambda=61, t_{\lambda}=276$ and $i=224$, we have that $a(M)=a_{224}^{(61)}=121895$. Hence, combining the conclusion of Lemma 3 and the inequality (51), we get

$$
\alpha^{n_{1}}<16.62 \times 121897 n_{2}\left(k_{2}-k_{1}\right)<2.02 \times 10^{314},
$$


so $n_{1} \leq 1503$. Hence, we obtain that $n_{1} \leq 7019$ holds in all cases $\left(v=n_{1}, \lambda<\chi\right.$ or $\left.\lambda=\chi\right)$. By the inequality (17), we have that

$\log \delta \leq k_{1} \log \delta \leq\left(n_{1}+1\right) \log \alpha<3378$.

By considering the second inequality in (25), we can conclude that $n_{2} \leq 1.0 \times 10^{38}\left(\log n_{2}\right)^{2}$, which immediately yields $n_{2}<3.5 \times 10^{40}$, by a simple application of Lemma 6 . We summarise the first cycle of our reduction process as follows:

$$
n_{1} \leq 7019 \text { and } n_{2} \leq 3.5 \times 10^{40} \text {. }
$$

From the above, we note that the upper bound on $n_{2}$ represents a very good reduction of the bound given in Lemma 8. Hence, we expect that if we restart our reduction cycle with the new bound on $n_{2}$, then we get a better bound on $n_{1}$. Thus, we return to the inequality (45) and take $M:=3.5 \times 10^{40}$. A computer search in Mathematica reveals that

$$
q_{86}>M>n_{2}>k_{2}-k_{1} \text { and } a(M):=\max \left\{a_{i}: 0 \leq i \leq 86\right\}=a_{21}=29,
$$

from which it follows that $\lambda \leq 400$. We now return to (47) and we put $X:=5.25 \times 10^{41}$ and $C:=(10 X)^{5}$ and then apply the LLL algorithm in Lemma 5 to $\lambda \in[1,400]$. After a computer search, we get

$$
1 \times 10^{-172}<\min _{1 \leq \lambda \leq 400}\left|\Gamma_{4}\right|<16.62 n_{2} \alpha^{-v}
$$

then $v \leq 1022$. By continuing under the assumption that $n_{j}-m_{j}=v \leq 1022$, we return to $(48)$ and put $X:=5.25 \times 10^{41}, C:=(10 X)^{9}$ and $M:=3.5 \times 10^{40}$ for the case $\lambda<\chi$ and $\lambda=\chi$. After a computer search, we confirm that

$$
2 \times 10^{-344}<\min _{\substack{1 \leq \lambda \leq 400 \\ \lambda+1 \leq \chi \leq 1022}}\left|\Gamma_{5}\right|<16.62 n_{2} \alpha^{-n_{1}}
$$

gives $n_{1} \leq 1844$, and $a(M)=a_{55}^{(117)}=30400$, leads to $n_{1} \leq 415$. Hence, in both cases $n_{1} \leq 1844$ holds. This gives $n_{2} \leq 4.2 \times 10^{38}$ by a similar procedure as before.

We record what we have proved.

Lemma 9. Let $\left(k_{i}, n_{i}, m_{i}\right)$ be a solution to $x_{i}=F_{n_{i}}+F_{m_{i}}$, with $0 \leq m_{i} \leq n_{i}$ for $i=1,2$ and $1 \leq k_{1}<k_{2}$ and where $d \neq 5 \square$, then

$$
\max \left\{k_{1}, m_{1}\right\} \leq n_{1} \leq 1844 \text { and } \max \left\{k_{2}, m_{2}\right\} \leq n_{2} \leq 4.2 \times 10^{38} \text {. }
$$

\subsection{The final reduction}

Returning back to (12) and (14) and using the fact that $\left(x_{1}, y_{1}\right)$ is the smallest positive solution to the Pell equation (1), we obtain

$$
\begin{aligned}
x_{k} & =\delta^{k}+\sigma^{k}=\left(\frac{x_{1}+y_{1} \sqrt{d}}{2}\right)^{k}+\left(\frac{x_{1}-y_{1} \sqrt{d}}{2}\right)^{k} \\
& =\left(\frac{x_{1}+\sqrt{x_{1}^{2} \mp 4}}{2}\right)^{k}+\left(\frac{x_{1}-\sqrt{x_{1}^{2} \mp 4}}{2}\right)^{k}:=P_{k}^{ \pm}\left(x_{1}\right) .
\end{aligned}
$$

Thus, we return to the Diophantine equation $x_{k_{1}}=P_{n_{1}}+P_{m_{1}}$ and consider the equations

$$
P_{k_{1}}^{+}\left(x_{1}\right)=F_{n_{1}}+F_{m_{1}} \text { and } P_{k_{1}}^{-}\left(x_{1}\right)=F_{n_{1}}+F_{m_{1}} \text {, }
$$

with $k_{1} \in[1,1844], m_{1} \in[0,1844]$ and $n_{1} \in\left[m_{1}+2,1844\right]$.

Besides the trivial case $k_{1}=1$, with the help of a computer search in Mathematica on the above equations in (53), we list the only nontrivial solutions in Table 1 . We also note that $3+2 \sqrt{2}=(1+\sqrt{2})^{2}$, so these solutions come from the same Pell equation when $d=2$. 


\begin{tabular}{|ccccc|}
\hline \multicolumn{5}{|c|}{$P_{k_{1}}^{+}\left(x_{1}\right)$} \\
\hline$k_{1}$ & $x_{1}$ & $y_{1}$ & $d$ & $\delta$ \\
\hline 2 & 6 & 4 & 2 & $3+2 \sqrt{2}$ \\
2 & 4 & 2 & 3 & $2+\sqrt{3}$ \\
2 & 16 & 6 & 7 & $8+3 \sqrt{7}$ \\
2 & 5 & 1 & 21 & $(5+\sqrt{21}) / 2$ \\
2 & 25 & 3 & 69 & $(25+3 \sqrt{69}) / 2$ \\
2 & 40 & 2 & 399 & $20+\sqrt{399}$ \\
\hline
\end{tabular}

\begin{tabular}{|ccccc|}
\hline \multicolumn{5}{c|}{$P_{k_{1}}^{-}\left(x_{1}\right)$} \\
\hline$k_{1}$ & $x_{1}$ & $y_{1}$ & $d$ & $\delta$ \\
\hline 2 & 2 & 2 & 2 & $1+\sqrt{2}$ \\
2 & 10 & 2 & 26 & $5+\sqrt{26}$ \\
2 & 12 & 2 & 37 & $6+\sqrt{37}$ \\
2 & 40 & 2 & 401 & $20+\sqrt{401}$ \\
\hline
\end{tabular}

Table 1. Solutions to $P_{k_{1}}^{ \pm}\left(x_{1}\right)=F_{n_{1}}+F_{m_{1}}$

From the above tables, we set each $\delta:=\delta_{t}$ for $t=1,2, \ldots 9$. We then work on the linear forms in logarithms $\Gamma_{1}$ and $\Gamma_{2}$, in order to reduce the bound on $n_{2}$ given in Lemma 9. From the inequality (23), for $(k, n, m):=\left(k_{2}, n_{2}, m_{2}\right)$, we write

$$
\left|k_{2} \frac{\log \delta_{t}}{\log \alpha}-n_{2}+\frac{\log (\sqrt{5})}{\log \alpha}\right|<\left(\frac{4}{\log \alpha}\right) \alpha^{-\left(n_{2}-m_{2}\right)},
$$

for $t=1,2, \ldots 9$.

We put

$$
\tau_{t}:=\frac{\log \delta_{t}}{\log \alpha}, \quad \mu_{t}:=\frac{\log (\sqrt{5})}{\log \alpha} \quad \text { and } \quad\left(A_{t}, B_{t}\right):=\left(\frac{4}{\log \alpha}, \alpha\right) .
$$

We note that $\tau_{t}$ is transcendental by the Gelfond-Schneider's Theorem and thus, $\tau_{t}$ is irrational. We can rewrite the above inequality, (54) as

$$
0<\left|k_{2} \tau_{t}-n_{2}+\mu_{t}\right|<A_{t} B_{t}^{-\left(n_{2}-m_{2}\right)}, \quad \text { for } t=1,2, \ldots, 9 .
$$

We take $M:=4.2 \times 10^{38}$ which is the upper bound on $n_{2}$ according to Lemma 9 and apply Lemma 4 to the inequality (55). As before, for each $\tau_{t}$ with $t=1,2, \ldots, 9$, we compute its continued fraction $\left[a_{0}^{(t)}, a_{1}^{(t)}, a_{2}^{(t)}, \ldots\right]$ and its convergents $p_{0}^{(t)} / q_{0}^{(t)}, p_{1}^{(t)} / q_{1}^{(t)}, p_{2}^{(t)} / q_{2}^{(t)}, \ldots$. For each case, by means of a computer search in Mathematica, we find and integer $s_{t}$ such that

$$
q_{s_{t}}^{(t)}>2.52 \times 10^{39}=6 M \quad \text { and } \quad \epsilon_{t}:=\left\|\mu_{t} q^{(t)}\right\|-M \| \tau_{t} q^{(t)} \mid>0 .
$$

We finally compute all the values of $b_{t}:=\left\lfloor\log \left(A_{t} q_{s_{t}}^{(t)} / \epsilon_{t}\right) / \log B_{t}\right\rfloor$. The values of $b_{t}$ correspond to the upper bounds on $n_{2}-m_{2}$, for each $t=1,2, \ldots, 9$, according to Lemma 4. With the help of Mathematica we got that the maximum value of $n_{2}-m_{2}$ is 201 for $t \in[1,9]$. The results of the computation for each $t$ are recorded in Table 2 below.

\begin{tabular}{llcccc}
\hline$t$ & $\delta_{t}$ & $s_{t}$ & $q_{s_{t}}$ & $\varepsilon_{t}>$ & $b_{t}$ \\
\hline 1 & $1+\sqrt{2}$ & 81 & $4.51994 \times 10^{39}$ & 0.388126 & 194 \\
2 & $2+\sqrt{3}$ & 72 & $8.76409 \times 10^{40}$ & 0.225348 & 201 \\
3 & $8+3 \sqrt{7}$ & 76 & $1.32196 \times 10^{40}$ & 0.421692 & 196 \\
4 & $(5+\sqrt{21}) / 2$ & 80 & $6.12803 \times 10^{39}$ & 0.142135 & 197 \\
5 & $5+\sqrt{26}$ & 70 & $2.62621 \times 10^{39}$ & 0.158712 & 195 \\
6 & $6+\sqrt{37}$ & 89 & $3.06359 \times 10^{39}$ & 0.241184 & 194 \\
7 & $(25+3 \sqrt{69}) / 2$ & 68 & $2.75772 \times 10^{39}$ & 0.048435 & 197 \\
8 & $20+\sqrt{399}$ & 84 & $2.84745 \times 10^{39}$ & 0.399493 & 193 \\
9 & $20+\sqrt{401}$ & 80 & $4.10314 \times 10^{39}$ & 0.125005 & 196 \\
\hline
\end{tabular}

Table 2. First reduction computation results 
By replacing $(k, n, m):=\left(k_{2}, n_{2}, m_{2}\right)$ in the inequality (20), we can write

$$
\left|k_{2} \frac{\log \delta_{t}}{\log \alpha}-n_{2}+\frac{\log \left((\sqrt{5}) /\left(1+\alpha^{-\left(n_{2}-m_{2}\right)}\right)\right)}{\log \alpha}\right|<\left(\frac{4}{\log \alpha}\right) \alpha^{-n_{2}}, \text { for } t=1,2, \ldots, 9 .
$$

We now put

$$
\tau_{t}:=\frac{\log \delta_{t}}{\log \alpha}, \quad \mu_{t, n_{2}-m_{2}}:=\frac{\log \left((\sqrt{5}) /\left(1+\alpha^{-\left(n_{2}-m_{2}\right)}\right)\right)}{\log \alpha} \quad \text { and } \quad\left(A_{t}, B_{t}\right):=\left(\frac{4}{\log \alpha}, \alpha\right) .
$$

With the above notations, we can rewrite (56) as

$$
0<\left|k_{2} \tau_{t}-n_{2}+\mu_{t, n_{2}-m_{2}}\right|<A_{t} B_{t}^{-n_{2}}, \quad \text { for } t=1,2, \ldots 9 .
$$

We again apply Lemma 4 to the above inequality (57), for

$$
t=1,2, \ldots, 9, \quad n_{2}-m_{2}=1,2, \ldots, b_{t}, \quad \text { with } \quad M:=4.2 \times 10^{38} .
$$

We take

$$
\varepsilon=\varepsilon_{t, n_{2}-m_{2}}:=\left\|\mu_{t} q^{\left(t, n_{2}-m_{2}\right)}\right\|-M\left\|\tau_{t} q^{\left(t, n_{2}-m_{2}\right)}\right\|>0,
$$

and

$$
b=b_{t, n_{2}-m_{2}}:=\left\lfloor\log \left(A_{t} q_{s_{t}}^{\left(t, n_{2}-m_{2}\right)} / \epsilon_{t, n_{2}-m_{2}}\right) / \log B_{t}\right\rfloor .
$$

With the help of Mathematica, we obtain the results in Table 3.

\begin{tabular}{l|ccccccccc}
\hline$t$ & 1 & 2 & 3 & 4 & 5 & 6 & 7 & 8 & 9 \\
$\varepsilon>$ & 0.0019 & 0.0008 & 0.0006 & 0.0005 & 0.0017 & 0.0026 & 0.0016 & 0.0038 & 0.0071 \\
$b$ & 207 & 215 & 211 & 213 & 204 & 205 & 209 & 204 & 202 \\
\hline
\end{tabular}

Table 3. Final reduction computation results

Therefore, $\max \left\{b_{t, n_{2}-m_{2}}: t=1,2, \ldots, 9\right.$ and $\left.n_{2}-m_{2}=1,2, \ldots b_{t}\right\} \leq 215$.

Thus, by Lemma 4 , we have that $n_{2} \leq 215$, for all $t=1,2, \ldots, 9$, and by the inequality (18) we have that $n_{1} \leq n_{2}+2$. From the fact that $\delta^{k} \leq \alpha^{n+1}$, we can conclude that $k_{1}<k_{2} \leq 104$. Collecting everything together, our problem is reduced to search for the solutions for (16) in the following range

$$
1 \leq k_{1} \leq k_{2} \leq 110, \quad 0 \leq m_{1} \leq n_{1} \leq 220 \quad \text { and } \quad 0 \leq m_{2} \leq n_{2} \leq 220 .
$$

After a computer search on the equation (16) on the above ranges, we obtained the following solutions, which are the only solutions for the exceptional $d$ cases we have stated in Theorem 1:

For the +4 case:

$$
\begin{array}{ll}
(d=2) & x_{1}=6=F_{5}+F_{2}=F_{4}+F_{4}, \quad x_{2}=34=F_{9}+F_{0}=F_{8}+F_{7} ; \\
(d=3) & x_{1}=4=F_{4}+F_{2}=F_{3}+F_{3}, \quad x_{2}=14=F_{7}+F_{2} ; \\
(d=7) & x_{1}=16=F_{7}+F_{4}, \quad x_{2}=254=F_{13}+F_{8} ; \\
(d=21) & x_{1}=5=F_{5}+F_{0}=F_{4}+F_{3}, x_{2}=23=F_{8}+F_{3}, x_{3}=110=F_{11}+F_{8} .
\end{array}
$$

For the -4 case:

$$
\begin{array}{ll}
(d=2) \quad x_{1}=2=F_{3}+F_{0}=F_{2}+F_{2}, \quad x_{2}=6=F_{5}+F_{2}=F_{4}+F_{4}, \\
\\
x_{3}=14=F_{7}+F_{2}, \quad x_{4}=34=F_{9}+F_{0}=F_{8}+F_{7} ; \\
(d=26) \quad x_{1}=10=F_{6}+F_{3}=F_{5}+F_{5}, \quad x_{2}=102=F_{11}+F_{7} .
\end{array}
$$

This completes the proof of Theorem 1 . 


\section{Acknowledgements}

M. D. was supported by the Austrian Science Fund (FWF) grants: F5510-N26 - Part of the special research program (SFB), "Quasi-Monte Carlo Methods: Theory and Applications" and W1230 - "Doctoral Program Discrete Mathematics". M. D. would also like to thank his supervisor Professor Robert Tichy for the encouragement and support during his PhD studies in Graz. F. L. was also supported by grant CPRR160325161141 from the NRF of South Africa, RTNUM18 from the CoEMaSS at Wits and by grant no. 17-02804S of the Czech Granting Agency.

\section{References}

[1] Bravo E F, Gómez-Ruiz C A, and Luca F, X-coordinates of Pell equations as sums of two tribonacci numbers, Periodica Mathematica Hungarica, 77 (2018) 175-190

[2] Gómez C A and Luca F, Zeckendorf representations with at most two terms to $x$ coordinates of Pell equations, Science China Mathematics, (2018) To appear, https: //link. springer.com/article/10.1007/s11425-017-9283-6

[3] Bugeaud Y, Mignotte M, and Siksek S, Classical Classical and modular approaches to exponential Diophantine equations I. Fibonacci and Lucas perfect powers. Annals of Mathematics, 163 (2006) 969-1018

[4] Cohen H, Number Theory. Volume I: Tools and Diophantine Equations Graduate Texts in Mathematics 239 (2017), (Springer) pp. 58-63

[5] Ddamulira M, On the $x$-coordinates of Pell equations which are sums of two Padovan numbers, Preprint (2019)

[6] Ddamulira M and Luca F, On the $x$-coordinates of Pell equations which are $k$ generalized Fibonacci numbers, Preprint (2018)

[7] Dossavi-Yovo A, Luca F, and Togbé A, On the $X$-coordinates of Pell equations which are rep-digits, Publicationes Mathematicae Debrecen, 88 (2016) 381-399

[8] Dujella A and Pethô A, A generalization of a theorem of Baker and Davenport, The Quarterly Journal of Mathematics, 49(1998) 291-306

[9] Faye B and Luca F, On the $X$-coordinates of Pell equations which are repdigits, The Fibonacci Quarterly, 56 (2018) 52-62

[10] Gúzman S S and Luca F, Linear combinations of factorials and $s$-units in a binary recurrence sequence, Annales Mathemántiques du Québec, 38 (2014) 169-188

[11] Kafle B, Luca F, and Togbé A, On the $x$-coordinates of Pell equations which are Fibonacci numbers II, Colloquium Mathematicum, 149 (2017) 75-85

[12] Kafle B, Luca F, and Togbé A, $x$-Coordinates of Pell equations which are Tribonacci numbers II, Periodica Mathematica Hungarica, (2018) To appear

[13] Kafle B, Luca F, and Togbé A, X-coordinates of Pell equations which are Lucas numbers, Boletín de la Sociedad Matemática Mexicana, (2018) To appear

[14] Luca F, Montejano A, Szalay L, and Togbé A, On the $x$-coordinates of Pell equations which are Tribonacci numbers, Acta Arithmetica, 179 (2017) 25-35

[15] Luca F and Togbé A, On the $x$-coordinates of Pell equations which are Fibonacci numbers, Mathematica Scandinavica, 122 (2018) 18-30

[16] Matveev E M, An explicit lower bound for a homogeneous rational linear form in the logarithms of algebraic numbers. II, Izvestiya: Mathematics, 64 (2000) 1217-1269

[17] Rihane S S, Hernane M O, and Togbé A, The $x$-coordinates of Pell equations and Padovan numbers, Turkish Journal of Mathematics, 43 (2019) 207-223 